\title{
LA ENSEÑANZA DE DERECHOS HUMANOS EN LAS ESCUELAS: UNA MIRADA SOBRE LA POLÍTICA EDUCACIONAL EN EL EESTADO DE PERNAMBUCO, BRASIL
}

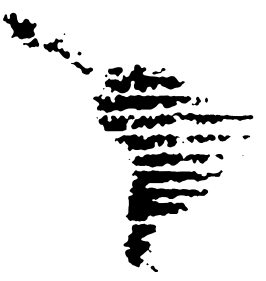

\section{HUMAN RIGHTS EDUCATION IN THE SCHOOLS: A LOOK AT THE EDUCATIONAL POLICYIN THE STATE OF PENAMBUCO / BRAZIL}

\author{
Celma Tavares*
}

Resumen

La educación en derechos humanos $(\mathrm{EDH})$, básica en el ámbito de una política educativa dirigida a la formación ciudadana, es un campo de conocimiento reciente en Brasil. En Pernambuco, el proceso de inclusión de la EDH en la red pública estatal de enseñanza se desarrolló de una forma más sistemática a partir del año 2007, convirtiéndose en referencia para el resto de los Estados brasileños. El objetivo de este texto consiste en analizar las características de esta iniciativa realizada entre 2007-2014, a partir de los datos recogidos en la Secretaria de Educación de Pernambuco. Se trata de un estudio documental con un abordaje metodológico cualitativo. En el análisis se identificaron dos fases en dicho proceso, donde las acciones de inclusión de la EDH alternaron la disciplinariedad y la transversalidad. Además, se mostró que en muchos aspectos la experiencia siguió las directrices de los documentos internacionales y nacionales del área, articulando las esferas cognitiva, ético-valórica y la de los comportamientos. Sin embargo, en cuanto proceso, se produjeron momentos de mayor y de menor presencia de estos parámetros. Por este motivo, ejes como la formación, la organización del currículo y la producción de materiales pedagógicos deben ser reforzados.

Palabras clave: educación en derechos humanos, política de la educación, formación ciudadana, enseñanza formal.

\footnotetext{
* Doctora en Derechos Humanos por la Universidad de Salamanca, España; profesora en la Maestría en Derechos Humanos de la Universidad Federal de Pernambuco, Brasil, vice-coordinadora e investigadora del Núcleo de Estudios y Pesquisas de Educación en Derechos Humanos, Diversidad y Ciudadanía de la misma universidad. Es también investigadora del Grupo de Investigación Derechos Humanos en Brasil del Centro de Estudios Brasileños de la Universidad de Salamanca, España y del Grupo de Investigación Dimensiones de los Derechos Humanos del Instituto Jurídico Portucalense, de la Universidad de Oporto, Portugal.
} 


\begin{abstract}
Human rights education (HRE) is a recent field of knowledge in Brazil. It is important in the context of citizenship-building education policies. In Pernambuco, the process of inclusion of HRE in the public network of state education was developed in a systematic way since 2007, and it become a model to other Brazilian States. The objective of this paper is to analyze the characteristics of this initiative conducted between 2007 and 2014, from data collected in the Ministry of Education of Pernambuco. This is a documentary study that makes use of a qualitative methodological approach. The analysis identified two stages in this process, where the actions of inclusion of HRE alternated disciplinarity with transversality. Furthermore, it was shown that in many respects the experience followed the guidelines of international and national documents in the field, linking cognitive areas, ethical and value-based dimensions and behaviors. However, in terms of process, there were times of greater and lesser presence of these parameters. For this reason, axes as training, curriculum organization and production of teaching materials should be reinforced.
\end{abstract}

Keywords: human rights education, educational policy, citizenship training; formal training.

Educar en derechos humanos tiene como finalidad posibilitar un cambio de mentalidad y de postura social que fomente la construcción de una cultura de respeto hacia tales derechos. Desde la Declaración Universal de los Derechos Humanos (1948) y con un énfasis más decidido a partir del Plan de Acción de Viena (1993), la comunidad internacional considera que la EDH forma parte del derecho a la educación, poniendo de relieve de manera continuada su consenso en torno a la radical importancia de esta en la consecución y realización de los demás derechos.

En Brasil, desde el periodo de redemocratización, la EDH se consolida en los procesos educativos y se integra progresivamente en la agenda gubernamental, en especial a partir de la segunda mitad de los años noventa. Se han dado así pasos importantes en el desarrollo de la EDH, entre los que destaca la elaboración de instrumentos normativos y la implementación de un conjunto de acciones. Apoyando esta conclusión, un estudio del Instituto Interamericano de Derechos Humanos (IIDH) muestra avances significativos en Brasil en comparación con los demás países del ámbito latinoamericano (Rodino, 2010).

En este contexto, el Estado de Pernambuco, ubicado en la región noreste de Brasil, destaca a nivel nacional gracias a una iniciativa desarrollada desde el año 2007, que superó el carácter de acción puntual, incorporando una estructura oficial y diversos mecanismos de acción dentro de la Secretaria de Educación de Pernambuco (SE/PE). Esto permitió ampliar el alcance de la iniciativa a toda la red de enseñanza, con 1.100 escuelas, integrando la EDH en el currículo y sus los proyectos político-pedagógicos. Por la singularidad de dicha experiencia, este artículo tiene por objetivo analizar la labor realizada por la SE/PE en el periodo 2007-2014; con el propósito de mostrar de qué 
modo sus planteamientos y actuaciones en torno a la educación vienen desarrollándose, y si estos se ajustan a los criterios teórico-metodológicos de dicha área, propios de la educación en derechos humanos.

\section{Política educacional, derechos humanos y EDH}

Un estudio sobre la inclusión de la EDH en la enseñanza formal demanda tanto un abordaje, aunque sea de forma breve, en torno a la comprensión de las políticas educacionales y de derechos humanos así como un recorrido por la construcción conceptual y normativa de la EDH.

La política educacional, según Höfling (2001, p. 31), es una "política pública social de responsabilidad del Estado -y sin embargo, no solo pensada por sus organismos". Aquí, de hecho, se entiende la política educacional de una "forma relacional" y "política" (Hage, 2011, p.75). Dicha comprensión implica la necesidad de situar esta política en el contexto de un Estado capitalista; es decir, considerar que la dinámica de realización está fuertemente marcada por el grado de participación popular y de desarrollo de las fuerzas productivas y de las relaciones de producción en determinada formación social (Neves, 1994, p. 16).

Además, uno de los aspectos a destacar en la formulación de las políticas educacionales en Brasil es su orientación "por la lógica de la discontinuidad/ continuidad, por carencia de planificación de largo plazo y por políticas de gobierno" (Dourado, 2010, p. 681). Esto conlleva un progresivo desmantelamiento en la elaboración y realización de acciones significativas en el campo de la educación en derechos humanos; dicha lógica condiciona las políticas de formación de ciudadanos(as), dificultando el desarrollo de una educación entendida "como factor de realización de la ciudadanía" (Libâneo, Oliveira y Toschi, 2012, p. 133).

En esta perspectiva, la materialización de una ciudadanía activa (Benevides, 1991) requiere el conocimiento sobre los derechos y la formación de valores y actitudes para su práctica; es decir, demanda procesos de formación continua, como políticas de Estado, y no solamente políticas de gobierno o proyectos aislados.

Los derechos humanos, a su vez, deben ser comprendidos como aquellos que garantizan la dignidad de la persona. La dignidad es "una descripción de las dimensiones de nuestra condición, el fundamento de nuestra ética pública, porque acota el ámbito de su acción, para realizar el proyecto en qué consiste el ser humano" (Peces-Barba, 2003, p. 50). Así, estos derechos constituyen prerrogativas básicas del ser humano y en los Estados democráticos deben estar positivados, si bien, aún sin formar parte de las leyes, son legítimamente exigibles (Silva y Tavares, 2011, p.16).

En Brasil, el inicio del proceso de institucionalización del Derecho Internacional de los Derechos Humanos ocurrió 
en el contexto de la redemocratización, momento en el que se ratificaron la mayoría de los tratados. Fue igualmente en este periodo cuando las políticas educacionales se articularon con las políticas de derechos humanos, promoviendo el desarrollo de acciones de EDH a nivel nacional. Este movimiento respondió a una estructuración interna por parte de la sociedad civil brasileña, a lo que se sumó un contexto internacional propicio, iniciado con la Declaración Universal, donde ya se destacaba el papel de la educación en el fomento del respeto a los derechos humanos.

De esta forma, la EDH ha ido ampliando su relevancia en foros internacionales, como la Conferencia Mundial de Derechos Humanos. Para consolidar el espacio de esta práctica se estableció el Decenio de las Naciones Unidas para la Educación en la Esfera de los Derechos Humanos, entre 1995-2004 (Naciones Unidas, 1994) y se elaboró el Programa Mundial para la Educación en Derechos Humanos (Naciones Unidas, 2004), iniciado en 2004 y con vigencia hasta 2014. Dentro del sistema interamericano destacan el Protocolo de San Salvador (OEA, 1988) y el Pacto Interamericano por la Educación en Derechos Humanos (OEA, 2010).

A pesar de ser una temática reciente en el contexto brasileño, la EDH cuenta con un cuadro normativo-institucional importante, como la Constitución Federal de 1988 (Brasil, 2011), la Ley de Directrices de la Educación (Brasil, 1996a), el Programa Nacional de
Derechos Humanos (Brasil, 1996b, 2002, 2009), y una oficina para el desarrollo de las acciones de EDH en el Ministerio de Educación y en la Secretaria de Derechos Humanos de la Presidencia de la República (SDH). En el campo de los documentos específicos, destaca el Plan Nacional de Educación en Derechos Humanos (PNEDH), elaborado por el Comité Nacional de Educación en Derechos Humanos, cuya primera versión data de 2003. Este plan se debatió en todo Brasil con el objetivo de perfeccionar su contenido, divulgándose en el año 2006 su versión final.

De acuerdo con el PNEDH la educación en derechos humanos es entendida como:

un proceso sistemático y multidimensional que orienta la formación del sujeto de derechos, articulando las siguientes dimensiones: a) aprehensión de conocimientos históricamente construidos sobre derechos humanos y su relación con los contextos internacional, nacional y local; b) afirmación de valores, actitudes y prácticas sociales que expresen la cultura de los derechos humanos en todos los espacios de la sociedad; c) formación de una conciencia ciudadana capaz de hacerse presente en los ámbitos cognitivo, social, ético y político; d) desarrollo de procesos metodológicos participativos y de construcción colectiva, utilizando un lenguaje y materiales didácticos contextualizados; e) fortalecimiento de prácticas 
individuales y sociales que generen acciones e instrumentos a favor de la promoción, la protección y la defensa de los derechos humanos, así como de la reparación de las violaciones de los mismos (Brasil, 2008, p. 25).

El Plan Nacional se estructura en cinco ejes: educación básica; educación superior; educación no formal; educación de profesionales del sistema de justicia y seguridad; y educación y medios de comunicación. Con este documento se definieron los principios y las directrices de las acciones gubernamentales para las políticas educacionales de derechos humanos.

El elemento más reciente en este cuadro normativo-institucional es la homologación, en el año 2012, de las Directrices Nacionales para la Educación en Derechos Humanos (DNEDH) por parte del Consejo Nacional de Educación, órgano del Ministerio de Educación, en forma de Parecer y de Resolución (Brasil, 2012a, 2012b). Entre otros aspectos, el documento, de carácter vinculante, establece la EDH como uno de los "ejes fundamentales del derecho a la educación" y señala la responsabilidad de los sistemas de enseñanza en la materialización de la $\mathrm{EDH}$. En este sentido, es importante subrayar que, a pesar de que la escuela no es el único lugar donde los conocimientos sobre derechos humanos se construyen, es donde se pueden hacer presentes de forma más sistemática (Brasil, 2012a, p.13).
A partir del el PNEDH se han venido realizando un conjunto de acciones en el ámbito de la educación básica. A fin de lograr la inclusión de la EDH en la red pública de enseñanza el Ministerio de Educación, en 2010, fomentó la elaboración de Planes de Acción en EDH para cada una de las escuelas por parte de los Estados. En líneas generales, los planes presentaron como objetivo el desarrollo de una educación basada en la formación humana y en una cultura de derechos humanos.

A pesar de la importancia de esta iniciativa, es necesario señalar que la actuación de las secretarias de educación en los diferentes Estados respecto a la EDH varía de acuerdo con el grado de institucionalización real en sus estructuras formales y también según su disponibilidad para transformar las estrategias en políticas públicas educacionales concretas. En este sentido, una investigación sobre la situación de la EDH en los Estados de Brasil, a partir de la elaboración de dichos planes, reveló que el trabajo, si bien está en desarrollo, aún se encuentra en su fase inicial y no se está dirigiendo a todo el sistema de enseñanza (Silva y Tavares, 2013).

El hecho diferencial dentro del panorama que se acaba de exponer se encuentra en Pernambuco, cuya experiencia en la inclusión de la EDH en la red pública de enseñanza logró el Premio Nacional de Educación en Derechos Humanos de la SDH en 2008. 


\section{El trayecto del estudio: metodología y diseño}

El estudio realizado sobre la experiencia de Pernambuco consiste en una investigación documental, cuyo abordaje toma como base la metodología cualitativa (Ludke y André, 1986; Ghedin y Franco, 2008; Gil, 1994). Así, se manejaron un conjunto de documentos elaborados por la SE/PE en el ámbito de la EDH: decretos, instrucciones normativas, orientaciones pedagógicas e informes (que se encuentran en las referencias bibliográficas). Este criterio metodológico se justifica por el hecho de que los documentos reflejan las actitudes y creencias de las personas e instituciones que los producen, revelándose como una herramienta fundamental de acercamiento a su proceso de construcción y a las directrices y pautas que marcaron dicho proceso.

La valoración del material citado se realizó mediante el análisis de contenido (Bardin, 1977), ya que este proporciona las mejores técnicas para el examen de documentos escritos tales como los documentos educativos que aquí se abordan. En dicho análisis se han observado las reglas de homogeneidad, exhaustividad, exclusividad, objetividad y adecuación. Con el objetivo de analizar el proceso implementado en el Estado de Pernambuco, dos preguntas principales guiaron este estudio:

¿Cómo se está desarrollando la política de EDH en Pernambuco?
¿Sigue la política de EDH en Pernambuco las directrices teórico-metodológicas básicas en torno a educación y derechos humanos?

De esta manera, el camino metodológico tomado fue el siguiente:

- Selección de los documentos significativos elaborados en el periodo 2007-2014 referentes a la configuración de un cuadro normativo-institucional para la inclusión de la EDH en el sistema educativo de Pernambuco.

- Definición de elementos orientadores para la interpretación de los datos: a) estructura específica para el área; b) currículo; c) formación; d) material pedagógico; h) otras acciones.

- Explotación del material a partir de las categorías para efectuar el análisis de significados y la interpretación de los datos.

- Cotejo de los datos con algunas de las directrices teórico-metodológicas básicas de la EDH: a) concepto en consonancia con la normativa vigente; b) diseño curricular que contenga la visión de derechos humanos; c) formación con contenidos y metodologías constructoras del sujeto de derecho; d) acciones que no la reduzcan a momentos puntuales (Candau, 2008; Magendzo, 2010).

Por último, es importante señalar que los datos presentados a lo largo de este artículo forman parte de la 
investigación "Educación en Derechos Humanos en la Enseñanza Formal: un análisis de la política educacional de Pernambuco", desarrollada entre 2012-2015, y que se realizó con la financiación de la Fundación de Amparo a la Ciencia y Tecnología del Estado de Pernambuco.

\section{La EDH en Pernambuco}

Sin olvidar las políticas realizadas durante los años ochenta y noventa del siglo pasado (Silva, 2000), años en los que se realizaron acciones destinadas a articular la enseñanza y la formación ciudadana, fue en el 2007 cuando se inició de forma más amplia y sistemática la inclusión de la EDH en la red pública de enseñanza en Pernambuco. Esta iniciativa partió del programa de gobierno para el periodo 2007-2010, que estableció como principio orientador de su política educacional la educación para la ciudadanía (Frente Popular de Pernambuco, 2006). A este respecto es importante anotar que las políticas educacionales son declaraciones de compromiso y que para su eficaz desarrollo "es necesario contar con declaraciones de políticas explícitas y con una estrategia coherente de ejecución que, entre otras cosas, incluya una definición clara de las medidas, los mecanismos, las responsabilidades y los recursos" (Naciones Unidas, 2006, p. 47).

En el ámbito de la SE/PE, según mostraron los datos analizados, el proceso de inclusión de la $\mathrm{EDH}$ en la red pública de enseñanza entre 2007-2014 se puede dividir en dos fases: la primera, que abarca desde el año 2007 hasta el 2010; y la segunda que se inicia en el 2011, continuando vigente en la actualidad.

En líneas generales, en la primera fase, que englobó a toda la red de enseñanza de la formación básica, la propuesta de inserción de la EDH se realizó mediante una asignatura de Derechos Humanos, que era optativa, junto con la presencia transversal de contenidos en derechos humanos en los proyectos político-pedagógicos de las escuelas. En la segunda fase se optó por trabajar dichos contenidos solo de forma transversal en el total de las escuelas de enseñanza fundamental y en la mayoría de las escuelas de bachillerato, conocidas como escuelas regulares, donde el estudiantado asiste a clase en un solo turno; es decir, o por la mañana o por la tarde. La excepción dentro de esta segunda fase se encuentra en las 300 escuelas de bachillerato que funcionan a tiempo completo (mañana y tarde), conocidas como escuelas de referencia, y que en 2012 incluyeron en su currículo la asignatura obligatoria de Derechos Humanos. Es importante señalar que la responsabilidad sobre las escuelas de referencia y sobre las escuelas regulares recae en áreas diferentes dentro de la SE/PE.

La primera gran acción en el periodo 2007-2010 consistió en la reformulación de la estructura organizacional de la SE/PE. El Decreto N.o 30.362, de 17 de abril de 2007 (Pernambuco, 2007) creó la Gerencia de Políticas de Educación en Derechos Humanos 
(GEDH) dentro de la estructura de la SE/PE, inexistente hasta entonces. Otra gran acción fue la actualización del currículo a través de la inclusión de la asignatura de Derechos Humanos, junto con la promoción de un debate a nivel estatal con el fin de decidir de forma conjunta la forma de situar a la $\mathrm{EDH}$ como fundamento de los proyectos político-pedagógicos de las escuelas.

La GEDH se creó con la competencia de formular, ejecutar y evaluar la política de educación en derechos humanos de la SE/PE (Pernambuco, 2007). Para ello, debería coordinar sus actuaciones con otros sectores, realizar acciones de formación, elaborar materiales pedagógicos, realizar el seguimiento de las acciones, etc. (Pernambuco. GEDH, 2007). La existencia de un sector específico para la EDH, además de señalar las condiciones para su desarrollo, evidenció una voluntad institucional real, como razona el IIDH, puesto que sus funciones u objetivos tienden a incluir una propuesta de acciones y planes en la materia (IIDH, 2006).

El trabajo realizado por la GEDH, de acuerdo una vez más con el análisis de los datos realizado, se llevó a cabo utilizando el concepto de EDH explicitado en el PNEDH, comprendiéndola como un proceso sistemático y multidimensional que orienta la formación del sujeto de derecho. Este es un punto importante ya que una de las dificultades más significativas en el proceso de EDH es la propia polisemia del término, que puede generar opciones de trabajo equivocadas o puntuales, como por ejemplo, suponer que con la trasmisión de conocimiento sobre derechos humanos la EDH está presente (CANDAU, 2008). Así, es fundamental establecer qué se entiende por $\mathrm{EDH}$ y qué se pretende alcanzar. En este sentido, no se puede permitir que la EDH se sustituya por otras expresiones "más fáciles de ser asumidas, como la educación cívica o la educación democrática, o que se restrinja la $\mathrm{EDH}$ a una educación en valores, inhibiendo su carácter político" (CANDAU, 2008, p. 289).

La implantación de un currículo unificado para la enseñanza fundamental y el bachillerato se hizo por medio de la Instrucción Normativa № 03/2008, de modo que se garantizase la equidad en la oferta de las asignaturas y su carga lectiva (Pernambuco. Sede, 2008). Antes del año 2007 no había uniformidad respecto a estas cuestiones, limitando el acceso de los alumnos y las alumnas a un contenido común dentro de toda la red pública de enseñanza. Con el nuevo currículo fue posible incluir el contenido de derechos humanos en forma de asignatura electiva/optativa. Otro documento de apoyo a este proceso fue la Base Curricular Común en las áreas de portugués y matemáticas que, entre otras cuestiones, señalaba que los conocimientos deben abarcar "la formación para la ciudadanía, entendida como la construcción del derecho a tener derechos" (Pernambuco, Secretaría de Educación, 2008, p. 11). 
A fin de reforzar el valor de la asignatura de Derechos Humanos, la GEDH elaboró un documento de orientación pedagógica, donde recogía la finalidad de la asignatura, sus posibles contenidos, varias sugerencias metodológicas y las referencias bibliográficas a seguir. El objetivo de la asignatura, descrito en el documento, buscaba fomentar

la comprensión de las bases conceptuales e históricas de los derechos humanos, de la reconstrucción histórica en el proceso de afirmación de estos derechos en la sociedad brasileña, despertando el interés en el debate y en la participación en cuestiones relativas a la ciudadanía y a la vivencia plena de los derechos, contribuyendo al desarrollo de las responsabilidades" (Pernambuco, GEDH, 2008a, p. 1).

En este mismo documento, la GEDH organizó la asignatura en cuatro etapas, con los siguientes temas y contenidos (Pernambuco, GEDH, 2008a, pp. 2-6):

\section{Unidad I - Tema: Derechos humanos}

- Fundamentos históricos de los derechos humanos

- Derechos civiles y políticos

- Derechos económicos y sociales

- Derechos de solidaridad

- La Declaración Universal de los Derechos Humanos
Unidad II - Tema: La evolución de los derechos humanos en Brasil

- La legislación y los derechos humanos en Brasil

- Movimientos sociales y derechos humanos en Brasil

- Los media y las diferentes formas de respeto y no respeto a los derechos humanos en Brasil

- Derechos de los discapacitados, de las personas mayores; de los niños(as) y adolescentes

\section{Unidad III - Tema: Prejuicio, racismo y desigualdad en Brasil}

- Racismo y prejuicio en Brasil

- La lucha de los indígenas y la violación de sus derechos

- "Quilombo" - espacio de resistencia de negros y negras

- La exclusión socioeconómica de la populación afrodescendiente en Brasil

- Conociendo las leyes: la Ley en contra del Racismo

Unidad IV - Tema: Equidad de género

- Concepto de género y relaciones de género

- Combate a la violencia contra la mujer 
- Las relaciones de género y el mundo del trabajo

- La Ley "Maria da Penha” (ley de combate a la violencia contra la mujer)

Este tipo de contenidos constituye uno de los aspectos fundamentales del trabajo de la EDH. De hecho, es necesario comprender que:

La educación en derechos humanos no se limita a la contextualización y a la explicación de las variables sociales, económicas, políticas y culturales que interfieren y orientan los procesos educativos. Va más allá de la contextualización, aunque esta sea imprescindible para la comprensión de su construcción. Forma parte de esta educación aprehender los contenidos que dan cuerpo a los derechos humanos, es decir: la historia, los procesos de evolución de las conquistas y de las violaciones de los derechos, las legislaciones, pactos y acuerdos que sostienen y garantizan los derechos, son contenidos que deben ser trabajados en el currículo básico [...] Los contenidos deben asociarse al desarrollo de valores, comportamientos éticos en la perspectiva de que el ser humano es siempre incompleto en términos de su formación. Por ser incompleto en cuanto ser social, datado, localizado, el ser humano posee la necesidad permanente de conocer, construir y reconstruir reglas de convivencia en sociedad (Silva, 2010, p. 49).
A su vez, el proceso formativo para las personas profesionales en el área de derechos humanos empezó en el año 2007, con una formación inicial sobre EDH destinada al equipo de la GEDH y con otro tipos de actividades, como seminarios y conferencias, y con la participación en los espacios de formación de la enseñanza media y fundamental. (Pernambuco, GEDH, 2010).

En el 2008 se inició una formación específica para 1.200 profesores(as) de la asignatura de Derechos Humanos, estructurada en módulos, realizándose también el 2009 y el 2010. Al mismo tiempo, se llevaron a cabo otras acciones de formación en áreas vinculadas a la GEDH, tales como la educación ambiental, la educación indígena y la educación étnico-racial, etc. (Pernambuco, GEDH, 2010). A partir del 2010, la formación pasó a dirigirse a los(as) educadores(as) de apoyo, quienes asisten al profesorado en la planificación de las actividades escolares.

Para la formación específica, la GEDH elaboró una propuesta pedagógica con el objetivo de "ofrecer instrumentos teórico-metodológicos necesarios para la realización de prácticas pedagógicas en derechos humanos" (Pernambuco, GEDH, 2008b, p. 1). Dicha formación tuvo una carga lectiva de 384 horas organizadas en encuentros mensuales, seminarios, vivencias de prácticas pedagógicas en las escuelas y en la elaboración de un plan de acción. Los contenidos se abordaron desde los siguientes ejes temáticos: a) Introducción a la 
historia de los derechos humanos y sus aspectos filosóficos; b) Las diversas caras de la violencia contra la mujer y los derechos de niños(as) y adolescentes; c) Currículo, derechos humanos y práctica docente; d) EDH: repensando la diferencia; e) Movimientos sociales y derechos humanos; f) Derechos humanos en el siglo XXI: retos y perspectivas; y h) Práctica pedagógica en EDH.

La metodología, según la propuesta citada, se basó en la interdisciplinaridad, en la contextualización y en la problematización de los contenidos curriculares, utilizándose seminarios, grupos de estudio, talleres pedagógicos, debates, actividades culturales y foros de discusión. Es importante subrayar que las labores formativas en derechos humanos para las personas profesionales de la educación responden a una necesidad señalada en todos los documentos internacionales, siendo imprescindible para la implantación de políticas públicas de EDH.

Respecto a esta cuestión es necesaria una mirada más atenta a las características de la formación en derechos humanos, a cómo es pensada, organizada y realizada, puesto que las buenas prácticas en este proceso van posibilitar una praxis pedagógica en consonancia con la EDH. Esto porque, como afirma Magendzo (1994, p. 146)

Pretender aproximarse a este saber como un espectador observante, como un acumulador acrítico, como un receptor pasivo, es desconocer la naturaleza del saber de la democracia y de los derechos humanos. Irremediablemente se deberá incorporar a las instituciones formadoras de maestros que pretendan transferir este saber, una metodología consonante. De lo contrario se caerá en la superficialidad y en la inoperancia. Esto sin duda sería el peor servicio que pudiera hacérsele a la causa de la educación de los derechos humanos y de la formación de educadores para la vida democrática.

Entre el 2008 y el 2010 la SE/PE llevó a cabo igualmente otras acciones, como la creación del Premio Educación Ciudadana, con el objetivo de fomentar la producción científica sobre derechos humanos; la creación de Muestras Pedagógicas de Buenas Prácticas, que incluyó el eje de la EDH; y la inclusión de los contenidos de derechos humanos en las oposiciones para el profesorado, donde se inscribieron cerca de 150.000 personas.

Por otro lado, a partir del 2011, se produjo una serie de cambios respecto a la etapa anterior. En primer lugar, el programa de gobierno para el periodo 2011-2014 se centró en el principio de una educación de calidad, con el objetivo de mejorar la posición de Pernambuco en el Índice de Desarrollo de la Educación Básica del Ministerio de Educación (Frente Popular de Pernambuco, 2010). En segundo lugar, hubo la retirada, en 2011, de la asignatura optativa de Derechos Humanos de las escuelas regulares, por medio de la Instrucción Normativa №. 02/2011 (Pernambuco, Sede, 2011), con la 
orientación de que se trabajara su contenido de forma trasversal. Por último, se incluyó en el currículo de las escuelas de referencia, en 2012, de la asignatura obligatoria de Derechos Humanos.

En este punto es necesario aclarar que las formas de organización del currículo que incluyera la $\mathrm{EDH}$ se indicaron en las DNEDH de la siguiente manera: por la transversalidad, por la disciplinariedad y de manera mixta; mezclando las dos anteriores (Brasil, 2012a). Sin embargo, en el caso de los cambios referidos, llama la atención la toma de decisiones contrapuestas dentro de un mismo órgano; es decir, la mayor parte de las escuelas sería orientada a trabajar los contenidos de derechos humanos de forma transversal y en otras 300 escuelas el modelo sería el de la disciplinariedad. Oficialmente no hubo justificaciones para una u otra medida.

En este escenario es importante señalar que dichos cambios pueden ser perjudiciales para el desarrollo de los contenidos de derechos humanos. Primero porque muchas veces la compresión que se tiene de educación de calidad no se articula con la concepción de $\mathrm{EDH}$ y segundo porque la opción por la transversalidad puede generar una reducción en el abordaje de los contenidos de derechos humanos.

Dentro de la formación se produjeron también modificaciones. Esta pasó del modelo adoptado en 2008, basado en la formación continua específica, a un modelo temático con contenidos relacionados con los derechos humanos como educación ambiental, educación indígena, etc. (Pernambuco, GEDH, 2011).

Asimismo, en el año 2012 destacaron tres acciones: la divulgación de las Orientaciones Curriculares de EDH, los Foros Regionales de EDH y el Programa de Formación de Gestores(as). Las Orientaciones, organizadas en ocho ejes, como la garantía de la sostenibilidad socioambiental o la igualdad entre géneros y diversidad sexual, se distribuyó entre el profesorado de toda la red pública de enseñanza, con el objetivo de "proporcionar apoyos al trabajo pedagógico" (Pernambuco, Sede, 2012, p. 7). Sin embargo, aún no se ha realizado la formación en torno a la utilización del material ni tampoco se ha llevado a cabo un seguimiento para conocer cuántas escuelas lo han adoptado y cómo lo están aplicando.

Los Foros se realizaron en todo el Estado, con la participación de 4.000 personas. En ellos se quiso profundizar en la discusión sobre las experiencias en el área y sobre las DNEDH. Las propuestas resultantes de los debates se organizaron en cuatro temas: violencia; medioambiente; relaciones étnico-raciales; y niñez, juventud y vejez.

El Programa de Formación, dirigido a futuros(as) gestores(as), se desarrolló en once módulos, con una carga lectiva de 180 horas (Pernambuco, 2012). Algunos de estos incluyeron temas relacionados con los derechos humanos, como la gestión centrada en valores o la 
diferenciación entre valores y derechos humanos (Pernambuco. Secretaría de Educación, 2012).

También es importante matizar que entre 2011-2012 la SE/PE divulgó la mayor parte de las Orientaciones Teórico Metodológicas (OTM) para el currículo de la enseñanza fundamental y del bachillerato, cuyo objetivo era apoyar las prácticas pedagógicas en cada área específica. En algunas de las OTM, todas del área de humanidades, como historia, geografía o sociología, es posible identificar temáticas que se conectan con contenidos de derechos humanos. Sin embargo, es la OTM de Historia para la enseñanza fundamental la que establece directamente dicha conexión, gracias a contenidos tales como "Derechos y deberes de los niños"; "Pernambuco y su construcción histórica y desarrollo de los derechos humanos; "El proceso de democratización en Brasil" (Pernambuco, Secretaría de Educación, 2011).

A partir de 2013 hubo una reducción de las acciones en el campo de los derechos humanos. En este período se destacan las Orientaciones para la Educación Ambiental; la propuesta pedagógica para los Centros de Atención a Adolescentes; y la adopción de Parámetros Curriculares para la red de enseñanza. De esta forma se identifica un cambio en el perfil de las actividades, siendo actualmente más escasas y en áreas más específicas.

Respecto a los Parámetros Curriculares, es importante decir que igualmente se identifican temáticas que se conectan con contenidos de derechos humanos. Dicha articulación está presente de forma más sistemática en los Parámetros de Historia (Pernambuco, Secretaría de Educación, 2013a) y, en menor medida, en los Parámetros de Geografía (Pernambuco, Secretaría de Educación, 2013b) y en los Parámetros de Filosofía y Sociología (Pernambuco, Secretaría de Educación, 2013c). En las demás áreas, el abordaje se restringe a su contenido específico, como Matemática, Física, Química, etc.

El ámbito de los materiales didáctico-pedagógicos, a su vez, representa una laguna en el trabajo de EDH por la ausencia de materiales apropiados a las necesidades de este campo. Es decir, los materiales deben ser compatibles con los principios de los derechos humanos y traer las representaciones de los diferentes grupos, de acuerdo con las directrices del Programa Mundial (Naciones Unidas, 2006, p. 46). Por ello, la contribución de materiales como las OTM y los Parámetros, organizados por la SE/PE son importantes; sin embargo, su abordaje se restringe a las asignaturas de las ciencias humanas. Lo que incluso va en sentido contrario a la orientación dada por la SE/PE de transversalidad de los contenidos de derechos humanos.

\section{Conclusiones}

La importancia y necesidad de la incorporación de la EDH a la enseñanza formal se ha venido consolidando a través de los instrumentos propuestos para tal fin en los sistemas internacional e 
interamericano. Esto queda patente en la evaluación realizada por el IIDH cuando afirma que "la incorporación de contenidos pedagógicos de derechos humanos y democracia en la educación formal constituye una medida fundamental para establecer una cultura de reconocimiento y respeto de los derechos" (IIDH, 2005, p. 8).

En la realidad brasileña, la EDH se constituye como un campo de conocimiento y práctica recientes. Por ello es un área en construcción, que presenta un amplio abanico de posibilidades. A su vez, la experiencia de inserción de la EDH en la red pública de enseñanza de Pernambuco, como se ha señalado anteriormente, se diferencia del escenario nacional.

En este estudio, de acuerdo con el análisis realizado, se ha tratado de mostrar el proceso realizado por la SE/PE, caracterizado por dos fases distintas, donde se alternaron acciones de inclusión de la $\mathrm{EDH}$, por medio de la disciplinariedad y de la transversalidad, estableciendo para cada momento una lógica propia que orienta el conjunto de estrategias desarrolladas.

Con base a las categorías temáticas adoptadas en el trabajo ha sido posible examinar dicho proceso. Respecto a la primera, que es la estructura específica para el área, se destaca la creación de la GEDH. En el ámbito de la segunda, que trata del currículo, la SE/PE realizó su actualización con la oferta de asignatura de derechos humanos y con acciones para abordar los contenidos de derechos humanos de forma trasversal. Sobre la formación, que es la tercera categoría, esta presentó formatos diferenciados, que variaron según cada fase, con contenidos específicos o temáticos. En la esfera del material pedagógico, dichos materiales aportaron orientaciones para la inserción de contenidos de derechos humanos de forma directa o indirecta, pero no hubo el seguimiento de su aplicación en las escuelas y se restringieron al campo de las ciencias humanas. Para la quinta categoría, que engloba otras acciones, se identificaron distintos instrumentos destinados a la integración de las actividades de los sectores de la SE/PE, como la creación de una muestra pedagógica y un premio.

Además, según las directrices teórico-metodológicos de la EDH utilizadas en esta investigación, ha sido posible identificar hasta qué punto el proceso consideró disposiciones de los documentos internacionales y nacionales relativos a los derechos humanos y la educación, buscando conjugar las esferas cognitiva, ético-valórica y de las actitudes. Así se ha mostrado cómo el concepto de EDH utilizado siguió la normativa vigente o cómo el diseño curricular incluyó la perspectiva de los derechos humanos. Sin embargo, en cuanto proceso general, el cumplimiento de las directrices en el área de la EDH no fue completo. Es decir, se produjeron momentos de mayor y de menor presencia de estos parámetros, según las opciones políticas, pedagógicas y metodológicas adoptadas por la $\mathrm{SE} / \mathrm{PE}$ en cada fase. 
Por todo ello, ejes como la formación, la organización del currículo y la producción de materiales pedagógicos se deben reforzar. En especial porque los procesos de implantación de políticas públicas no siguen trayectorias lineales sino que presentan ritmos y movimientos diferenciados que necesitan consolidarse.

\section{Referencias}

Bardin, L.(1977). Análise de conteúdo. Lisboa: Edições 70.

Benevides, M.(1991). A Cidadania Ativa. São Paulo: Ática.

BRASIL (2011). Constituição de 1988. Constituição da República Federativa do Brasil: promulgada em 5 de outubro de 1988. In: Vade Mecum. 11.ed. São Paulo: Saraiva, 9-83.

Lei n. ${ }^{\circ}$ 9.394, de 20 de dezembro de 1996. [Lei de Diretrizes e Bases da Educação Nacional] (1996a) Estabelece as diretrizes e bases da educação nacional. Diário Oficial [da] República Federativa do Brasil, Poder Executivo, Brasília, DF, 23 dez.

Decreto $n .^{\circ} 1.904$, de 13 de maio de 1996. Programa Nacional de Direitos Humanos 1. (1996b) Revogado pelo Decreto n.음 4.229, de 13.5.2002. Brasília: SEDH/MJ.

Decreto n. .94 .229 , de 13 de maio de 2002. Programa Nacional de Direitos Humanos 2. (2002) Revogado pelo Decreto n. 7.037, de 2009. Brasília: SEDH/MJ.

Plan Nacional de Educación en Derechos Humanos. (2008). Brasília: MEC/SEDH.

Decreto n. ${ }^{\circ}$ 7.037, de 21 de dezembro de 2009. (2009). Aprova o Programa Nacional de
Direitos Humanos - PNDH-3 e dá outras providências. Brasília: SEDH/MJ.

Parecer CNE/CP n.ำ 8/2012. (2012a).Diretrizes Nacionais para a Educação em Direitos Humanos. Brasília: MEC/CNE.

Resolução CNE/CP n.․․ 01/2012, de 30 de maio de 2012. (2012b). Estabelece Diretrizes Nacionais para a Educação em Direitos Humanos. Brasília: MEC/CNE.

Candau, Vera (2008). Educação em direitos humanos: questões pedagógicas. In: Bittar, Eduardo (org.). Educação e metodologia para os direitos humanos. São Paulo, Quartier Latin, 285-298.

Dourado, Luiz Fernandes (2010). Avaliação do Plano Nacional de Educação 20012009: questões estruturais e conjunturais de uma política. Educação $\mathcal{E}$ Sociedade, 31(112), 677-705.

Frente Popular de Pernambuco (2006). Programa de governo Eduardo Campos: um novo Pernambuco 2007-2010. Recife.

. (2010). Programa de governo Eduardo Campos: o novo Pernambuco 2011 2014. Recife.

Ghedin, E. \& Franco, M. (2008). Questões de método na construção da pesquisa em educação. São Paulo: Cortez.

Gil, A.(1994). Métodos e técnicas de pesquisa social. São Paulo: Atlas.

Hage, S. (2011). Educação, escola e políticas educacionais na perspectiva dos estudos culturais críticos: a produção do senso comum e as disputas pela hegemonia. Cadernos de Educação, 38, 69-93. 
Höfling, E.(2001). Estado e políticas (públicas) sociais. Cadernos Cedes, 21(55), $30-41.0$ http://dx.doi.org/10.1590/ S0101-32622001000300003

IIDH (2005). III Informe Interamericano de la Educación en Derechos Humanos: desarrollo en la formación de educadores. San José, Costa Rica: Instituto Interamericano de Derechos Humanos.

(2006). IV Informe Interamericano de la Educación en Derechos Humanos: desarrollo y planificación nacional. San José, Costa Rica; Instituto Interamericano de Derechos Humanos.

Libâneo, J, Oliveira, J., Toschi, M. (2012). Educação escolar: políticas, estrutura e organização. São Paulo: Cortez.

Lüdke, M.; André, M. (1986). Pesquisa em educação: abordagens qualitativas. São Paulo: EPU.

Magendzo, A. (1994). Formación de profesores para una educación para la vida democrática y el respeto a los Derechos Humanos. En: Magendzo, A. (Org.) Educación en derechos humanos: apuntes para una nueva práctica. Santiago: $\mathrm{CN}$ RR-PIIE, 139-146.

(2010). Ideas-fuerza y pensamiento de la educación en derechos humanos en Iberoamérica. En: (org.). Pensamiento e ideas-fuerza de la educación en derechos humanos en Iberoamérica. Santiago: OIE/Orealc/Unesco.

Naciones Unidas (1994). Decenio de Nacionas Unidas para la Educación en la Esfera de los Derechos Humanos das Nações Unidas para Educação em Matéria de Direitos Humanos 1995-2004. Resolución n. 49/184.
(2004). Programa Mundial para la Educacioón en Derechos Humanos, Resolución n. 59/113-A.

Naciones Unidas (2006). Plan de Acción para la primera etapa del Programa Mundial para la Educación en Derechos Humanos.

Neves, L.(1994). Educação e política no Brasil de hoje. São Paulo: Cortez.

OEA (1988). Protocolo adicional à Convenção Americana sobre Direitos Humanos em matéria de direitos econômicos, sociais e culturais: Protocolo de San Salvador. Assinado na Assembleia Geral da OEA, 18. Período Ordinário de Sessões.

(2010). Pacto Interamericano por la Educación en Derechos Humanos. Aprobado en la cuarta sesión plenaria de la 40 Asamblea General de la OEA.

Peces-Barba, G. (2003). La dignidad de la persona desde la Filosofía del Derecho. Madrid: Dyckinson.

Pernambuco (2007). Decreto N. 30.362 de 17 de abril de 2007. Aprova o Regulamento da Secretaria de Educação e dá outras providências. Diário Oficial do Estado, Recife, Poder Executivo, 18 abr. 2007.

(2012). Decreto n. ${ }^{\circ} 38.103$, de 25 de abril de 2012. Regulamenta os critérios e procedi mentos para realização de processo de seleção para função de representação de diretor escolar e diretor adjunto das escolas estaduais, e dá outras providências. Diário Oficial do Estado, Recife, Poder Executivo, 19 maio 2012.

Pernambuco. GEDH (2007). Diretrizes, competências e atribuições. 
(2008a). Componentes curriculares. Recife: SE/GEDH.

(2008b). Proposta de formação continuada. Recife: SE/GEDH.

(2010). Relatório de atividades 20072010, dezembro de 2010. Recife: SE/ GEDH.

.(2011). Relatório de atividades 2011. Recife: SE/GEDH.

Pernambuco, Secretaria de Educação (2008). Base curricular comum para as redes públicas de ensino de Pernambuco: língua portuguesa. Recife: SE.

. (2011). Orientações teórico-metodológicas de história: ensino fundamental. Recife, SE.

. (2012). Construindo a excelência em gestão escolar: curso de aperfeiçoamento: Módulo II Gestão com foco na educação em valores, cultura de paz e sustentabilidade. Recife: SE.

. (2013a). Parâmetros curriculares de história: ensino fundamental e médio. Recife: SE.

. (2013b). Parâmetros curriculares de geografia: ensino fundamental e médio. Recife: SE.

. (2013c).Parâmetros curriculares de filosofia e sociologia: ensino médio. Recife: SE.

Pernambuco. SEDE. (2008). Instrução Normativa n. ${ }^{\circ}$ 03/2008, de 4 de março de 2008. Dispõe sobre a implantação/operacionalização das Matrizes Curriculares nas Escolas da Rede Estadual de Educação a partir do ano letivo de 2008. Diário Oficial do Estado, Recife, 4 mar. 2008.

(2010). Políticas de Educação do Estado de Pernambuco 2007-2010. Recife: SE/PE.

(2011). III Mostra de Experiências Pedagógicas bem-sucedidas na escola pública de Pernambuco. Recife: SE/PE.

(2012). Orientações curriculares de educação em direitos humanos. Recife: $\mathrm{SE} / \mathrm{PE}$.

Rodino, A. (2010). Progresos de la educación en derechos humanos en América Latina: balance de dos décadas (1990-2010). En: Curso Interdisciplinario en Derechos Humanos: La Educación en Derechos Humanos, 28, 2010, San José, Costa Rica. San José, Costa Rica: IIDH.

Silva, A. (2000). A escola pública e a formação da cidadania: limites e possibilidades. Tese (Doutorado em Educação) - Faculdade de Educação, Universidade de São Paulo, São Paulo.

(2010). Direitos humanos na educação básica: qual o significado? In: SILVA, Aida; TAVARES, Celma. Política e fundamentos da educação em direitos humanos. São Paulo: Cortez, 41-63.

Silva, A. \& Tavares, C. (2011). A cidadania ativa e sua relação com a educação em direitos humanos. Revista Brasileira de Política e Administração da Educação, 27, 13-24.

(2013). Educação em direitos humanos no Brasil: contexto, processo de desenvolvimento, conquistas e limites. Educação (Porto Alegre, impresso) 36(1), 50-58.

Recibido: 21/9/2015 • Aceptado: 25/2/2016 